\title{
YTH-60, a novel multikinase inhibitor, potently ameliorates lung inflammation and fibrosis in preclinical model
}

\author{
Liu Hongyao ${ }^{1}$, Wu Xiuli ${ }^{1}$, Liu Zhihao ${ }^{1}$, Gan Cailing $^{1}$, Wei Wei ${ }^{1}$, Su Xingping ${ }^{1}$, Wang \\ Liqun $^{1}$, Zhang Qianyu ${ }^{1}$, Tan Zui ${ }^{1}$, Yue Lin $^{1}$, Yao Yuqin ${ }^{1}$, Liang Ouyang ${ }^{1}$, Luoting Yu ${ }^{1}$, and \\ ye tinghong ${ }^{1}$ \\ ${ }^{1}$ Sichuan University
}

May 18, 2020

\begin{abstract}
BACKGROUND AND PURPOSE Idiopathic pulmonary fibrosis (IPF) is characterized by excess accumulation of extracellular matrix, is involved in many chronic diseases or injuries and greatly threatens human health. However, clinical drugs have unexpected side effects. The development of novel, less toxic drugs to treat pulmonary fibrosis remains an urgent need. EXPERIMENTAL APPROACH YTH-60 was developed via computer-aided drug design, de novo synthesis and high-throughput screening. The biochemical, pharmacodynamic and toxicological profifiles of YTH-60 were investigated using kinase and cell viability assays, a bleomycin-induced mouse pulmonary fibrosis model and a TGF- $\beta 1$ induced epithelial-mensenchymal transition in A549 cell. KEY RESULTS YTH-60 displayed marked antiproliferative activity in fibroblasts and A549 cells. YTH60 suppressed the TGF- $\beta 1$ induced protein expression of collagen type I and alpha smooth muscle actin ( $\alpha$-SMA) in vitro. Moreover, intraperitoneal administration of YTH-60 at a dose of 15 and $30 \mathrm{mg}-1 * \mathrm{~kg}-1 *$ day*-1 for 2 weeks effectively alleviated the degree of fibrosis in a bleomycin-induced mouse pulmonary fibrosis model without obvious side effects. Importantly, YTH60 demonstrated decent bioavailability $(\mathrm{F}=17.86 \%)$ and suitable eliminated half-life time (T1/2 $=8.03 \mathrm{~h})$. CONCLUSION AND IMPLICATIONS YTH-60, a novel multikinase inhibitor, shows therapeutic potential for treating pulmonary fibrosis, and warrants further investigation as a potential drug candidate.
\end{abstract}

\section{What is already known}

Nintedanib is a clinical drug for the treatment of pulmonary fibrosis, but there are some unexpected side effects.

\section{What this study adds}

- YTH-60, a novel multikinase inhibitor, displayed marked antiproliferative activity both in vitro and in vivo .

- YTH-60 might be a potential drug candidate with low toxicity and has better absorption than nintedanib.

\section{Clinical significance}

a novel multikinase inhibitor YTH-60 could be a potential drug candidate for treating pulmonary fibrosis.

\section{INTRODUCTION}

Idiopathic pulmonary fibrosis (IPF) is a chronic and devastating lung disease of unknown aetiology(Raghu et al.,2011). The hallmark of IPF is chronic alveolar epithelial injury, which results in fibroblast activation, myoblast proliferation and extracellular matrix (ECM) deposition, thereby destroying lung architecture(Datta, Scotton \& Chambers,2011). Gene therapy is a potential treatment; however, successfully applying it in 
clinical practice has been a formidable challenge(Luigi,2016; Somia \& Verma,2000). Currently, only two drugs approved by the FDA, pirfenidone and nintedanib, have been considered promising therapeutic agents for slowing the progression of the disease, mainly by changing forced vital capacity (FVC)(Karimi-Shah \& Chowdhury,2015; Noble et al.,2011). It is still far from satisfaction due to the adverse reactions (Noble et al.,2016; Richeldi et al.,2016) and the limited efficacy of current drugs in protecting against pulmonary fibrosis. Therefore, the identification of a novel therapeutic drug in preclinical studies is highly desired.

A plethora of mediators, including cytokines and chemokines, have been implicated in the process of lung fibrosis. Among these molecules, the transforming growth factor (TGF)- $\beta_{1}$ markedly induces epithelialmesenchymal transition (EMT) in alveolar epithelial cells (AECs) and triggers the differentiation of fibroblasts into myofibroblasts(Hinz, Phan, Thannickal, Galli, Bochaton-Piallat \& Gabbiani,2007). In addition, epidermal growth factor receptor (EGFR) and platelet-derived growth factor receptor (PDGFR) are highly expressed in pulmonary fibrosis. EGFR and PDGFR are members of the tyrosine kinase superfamily, and tyrosine kinase inhibitors (TKIs) can block fibrosis signals and reduce fibroblast activation(Ingram \& Bonner). Finally, fibroblast growth factor (FGF) signaling pathway could bean ideal target for anti-fibrotic therapeutic approaches(Lin, Chen, Pan, Xu, Hu \& Xu,2011). FGF signalling through its receptors FGFR1, FGFR2, FGFR3, or FGFR4 regulates cell fate, angiogenesis, immunity, and metabolism(Masaru \& Katoh.,2016). Because of the important role of these signaling pathways in pulmonary fibrosis, and nintedanib is a triple angiokinase inhibitor that simultaneously acts on FGFR, VEGFR and PDGFR. Therefore, our research group has been interested in the design, synthesis and biological evaluation of novel multikinase inhibitors as potential new antifibrosis agents.

Our aim was to develop a novel multikinase inhibitor that potently and specifically blocks the FGF/FGFR signaling cascade system with little or no toxicity. We independently developed a series of compounds that effectively inhibit TGF- $\beta_{1}$-induced total collagen accumulation in the NIH-3T3 cell line and migration of A549 cells. Among these compounds, YTH-60 showed outstanding kinase inhibitory activity, including FGFR1, FGFR2, FGFR3, FGFR4, Abl and VEGFRs. This compound has also shown antiproliferative activities against a panel of fibroblast cells. In this study, we used the clinical drug nintedanib as a positive control and evaluated the activity of YTH-60 in inhibiting lung fibrosisin vitro and in vivo. We induced lung fibrosis in mice with bleomycin treatment in vivo and induced EMT in A549 cells and other fibroblasts with TGF- $\beta_{1}$ treatment in vitro. We found that administration of YTH-60 protected mice against lung fibrosis, as evidenced by reduced pulmonary fibrosis and lung damage. Our data indicated that YTH-60 could be a therapeutic target for the treatment of pulmonary fibrosis.

\section{METHODS}

\subsection{Synthesis of the compound YTH-60}

All chemical solvents and reagents were purchased from commercial sources and used as received. Thin-layer chromatography was performed on $0.20 \mathrm{~mm}$ silica gel 60 F254 plates (Qingdao Haiyang Chemical, China) and visualized under UV light $(254 \mathrm{~nm}) .{ }^{1} \mathrm{H}$ NMR and ${ }^{13} \mathrm{C}$ NMR spectra were recorded on a Bruker Avance-400 spectrometer or Varian spectrometer with TMS as an internal standard. The purities of all final compounds were greater than $95 \%$. The synthesis procedure is shown in Fig. S1. ${ }^{1} \mathrm{H}$ NMR $\left(400 \mathrm{MHz}, \mathrm{DMSO}-d{ }_{6}\right) \delta$ $13.28(\mathrm{~s}, 1 \mathrm{H}), 8.28(\mathrm{~d}, \mathrm{~J}=8.4 \mathrm{~Hz}, 1 \mathrm{H}), 7.78(\mathrm{~d}, \mathrm{~J}=8.1 \mathrm{~Hz}, 2 \mathrm{H}), 7.59(\mathrm{~d}, \mathrm{~J}=2.1 \mathrm{~Hz}, 2 \mathrm{H}), 7.39-7.32(\mathrm{~m}$, $3 \mathrm{H}), 7.00(\mathrm{~d}, \mathrm{~J}=8.2 \mathrm{~Hz}, 2 \mathrm{H}), 3.98(\mathrm{~s}, 6 \mathrm{H}), 3.20(\mathrm{~s}, 3 \mathrm{H}), 2.96(\mathrm{~s}, 2 \mathrm{H}), 2.36(\mathrm{~s}, 4 \mathrm{H}), 2.25(\mathrm{~s}, 4 \mathrm{H})$, and $2.12(\mathrm{~s}$, $3 \mathrm{H})$.

\subsection{Drugs and regents}

3-(4,5-dimethyl-2-thiazolyl)-2,5-di-phenyl-2H-tetrazoliumbromide(MTT),Dimethyl sulfoxide (DMSO) were purchased from Sigma Chemical Co.(St. Louis, MO,USA). The antibodies against $\alpha$-SMA, collagen I, Ecadherin and Vimentin were acquired from Abcam (Cambridge, MA, USA). $\beta$-actin was purchased from ZSJQ-BIO Co. (Beijing, China). The FITC-conjugated secondary antibody was purchased from Santa Cruz Biotechnology (Santa Cruz, CA). 


\subsection{Molecular modeling}

The molecular docking studies were carried out using GOLD 5.0. The crystal structure of FGFR1 (PDB ID: 5UR1) was retrieved from the RCSB Protein Data Bank and chosen as the structure of the reference protein. The molecular structures of compounds were constructed YTH-60 using Chemdraw software and saved as SDF format files.

\subsection{Kinase profile assay and $\mathrm{IC}_{50}$ test}

The in vitro kinase enzymatic inhibition assays were performed by the Kinase Profiling Services provided by Eurofins (UK). The detailed protocol descriptions can be provided by the website (https://www.eurofinsdiscoveryservices.com ).

\subsection{Cell lines and cell culture}

The human cancer cell lines A549 and human pulmonary fibroblasts (HPF) used in this study were purchased from American Type Culture Collection (ATCC, Manassas, VA). NIH3T3 was acquired from the China Center for Type Culture Collection (CTCCC, Wuhan, China). The normal human liver cell line LO2 was obtained from the Cell Bank of the Chinese Academy of Science (Shanghai, China). The cells were cultured in DMEM or RPMI 1640 media containing $10 \%$ foetal bovine serum and $0.1 \%$ amikacin sulfate under humidified conditions with $5 \% \mathrm{CO}_{2}$ at 37 .

\subsection{Cell proliferation assay}

The cell viability of YTH-60 treatment were performed by MTT assay $(\mathrm{Xu}, 2013)$. Briefly, the exponentially growing cells were seeded in 96-well plates at a density of $2-5 \times 10^{3}$ cells/well and cultured for $24 \mathrm{~h}$. The cells were treated with various concentrations of YTH- $60(0,0.625,1.25,2.5,5,10 \mu \mathrm{M})$, after treatment for 24 $\mathrm{h}, 48 \mathrm{~h}$ and $72 \mathrm{~h}$, respectively, a volume of $20 \mu \mathrm{L}$ of $5 \mathrm{mg} \cdot \mathrm{mL}^{-1} \mathrm{MTT}$ solution was added to each well and incubation for $2-4 \mathrm{~h}$ at 37 . The medium was subsequently discarded and $150 \mathrm{~mL}$ of DMSO was added to each well to dissolve the formazan crystal produced by living cells. 5 min later, the 96 -well plates were read on Spectra MAX M5 microplate spectrophotometer (Molecular Devices) at $570 \mathrm{~nm}$ wavelength, the percentage of inhibition and $\mathrm{IC}_{50}$ values were then calculated. Each assay was replicated 3 times.

\subsection{Western blot analysis}

Samples of mouse lung tissues and cells were homogenized in RIPA buffer in the presence of a protease inhibitor cocktail. The protein concentrations were examined using the Lowry method and equalized before loading. Approximately $50 \mu \mathrm{g}$ of total protein was separated by SDS-PAGE and then transferred to polyvinylidene fluoride (PVDF) membranes. The membranes were blocked for $1 \mathrm{~h}$ at $37^{\circ} \mathrm{C}$ and incubated with specific primary antibodies overnight at $4{ }^{\circ} \mathrm{C}$. After washing with TBST $(20 \mathrm{mM}$ Tris- $\mathrm{HCl}$ at $\mathrm{pH} 7.4$, $150 \mathrm{mM} \mathrm{NaCl}, 0.05 \%$ Tween-20), the membranes were incubated with HRP-labelled secondary antibodies for $1 \mathrm{~h}$ and visualized with enhanced chemiluminescence reagents (Amersham).

\subsection{Immunofluorescence assay}

NIH-3T3 and HPF cells were cultured in 24-well plates (Axygen, U.S.), and treated with YTH-60 (2.5 $\mu \mathrm{M})$ and TGF $\beta_{1}$ (Millipore, U.S.) $\left(5 \mathrm{ng} \cdot \mathrm{mL}^{-1}\right)$ for $24 \mathrm{~h}$. The cells were successively washed (three times with phosphate-buffered saline), fixed with $4 \%$ paraformaldehyde (Beyotime, China) for $15 \mathrm{~min}$, permeabilized with 0.3\% Triton X-100 (Beyotime, China) for $20 \mathrm{~min}$ at RT, blocked for 30 min with 5\% BSA (Beyotime, China), and then incubated with specific primary antibodies against $\alpha$-SMA and E-cadherin overnight at 4. After a wash step, the cells were incubated with Alexa 594-labelled anti-mouse IgG (red) (Thermo, \#A-11005, 1:1000 dilution) for $1.5 \mathrm{~h}$ at RT, and the nuclei were stained with DAPI (Beyotime, China) for another $10 \mathrm{~min}$ in the dark at RT. Images were acquired using an LSM 510 laser confocal microscope (Zeiss confocal microscope, Germany).

\subsection{Wound healing assay}


A549 cells were grown on 6-well plates until they formed a confluent monolayer. A scratch was made using a sterile $2.0 \times 10^{-7} \mathrm{~m}^{3}$ micropipette tip, and the cells were washed with PBS and treated with TGF- $\beta_{1}$ or YTH60. Cell migration was tracked over time and imaged by phase contrast microscopy after $24 \mathrm{~h}$ of treatment. Each culture was imaged at 3 different locations. The percentage of the original width was estimated by measuring the width between the edges of the scratch in the distinctive area of each image using ImageJ software.

\subsection{Quantitative real-time PCR (qPCR)}

Total RNA was isolated using AXYGEN reagent according to the manufacturer's instructions. The concentration of RNA was determined, and cDNA was generated using total RNA with a reverse transcriptase kit. The sequences of primers are listed in Supplementary Table 1. PCR amplification was performed for 40 cycles as follows: denaturation at 94 for $60 \mathrm{~s}$ and primer annealing for $60 \mathrm{~s}$.

\subsection{Bleomycin-induced mouse model of pulmonary fibrosis}

Seven-week-old male C57BL/6 mice were purchased from HFK Bioscience Co. Ltd., Beijing, China. All animals were housed in a controlled environment and given ad libitum access to food and water. All animal experiments were approved by the Animal Experimental Ethics Committee of Kyung Hee University (permit number: KHUASP (SE)-16-033) and were performed according to the local guidelines.

Pulmonary fibrosis was induced using bleomycin treatment $\left(2 \mathrm{mg} \cdot \mathrm{kg}^{-1}\right)$. Male C57BL $/ 6$ mice were randomly divided into four groups: a control group (sham), a bleomycin group (vehicle), a BLM+nintedanib group (nintedanib, $20 \mathrm{mg} \cdot \mathrm{kg}^{-1}$ ) and a BLM+YTH-60 group. In the BLM+YTH-60 group, mice in two subgroups were treated with YTH-60 at a dose of 15 or $30 \mathrm{mg} \cdot \mathrm{kg}^{-1}$. On day 0 , the mice were lightly anaesthetized using $10 \%$ chloral hydrate, and BLM was dissolved in saline and administered intratracheally. To test the preventive effect, on day 2 after BLM challenge, YTH-60 and nintedanib were administered intraperitoneally daily for 13 days (inflammatory phase) to the mice. All mice were killed $24 \mathrm{~h}$ after the last injection, and the lungs were harvested for the assays described below.

\subsection{Analysis of bronchoalveolar lavage fluid}

After the animals were sacrificed, bronchoalveolar lavage fluid (BALF) was collected by washing the lung three times with $1 \mathrm{ml}$ of saline. The total live-cell count was performed by an ADVIA 2120i.

\subsection{Histopathological staining}

Left lungs were detached from the mice and immediately fixed with $4 \%$ paraformaldehyde before embedding with paraffin wax and routine processing. Serial paraffin sections $(3-5 \mu \mathrm{m})$ were prepared using a microtome, and deparaffinized tissue sections were stained with haematoxylin and eosin to evaluate morphological changes in the lungs. The severity of lung fibrosis was evaluated using Masson's trichrome staining. Masson's staining was carried out to differentiate collagen from other fibres by staining nuclei black, cytoplasm and muscles red, and collagen blue.

Ashcroft scoring was used to determine the severity of pulmonary fibrosis; each field was semi-quantitatively assessed, as described previously with minor modifications(Ashcroft, Simpson \& Timbrell). The criteria for grading fibrosis were as follows: Grade 0, normal lung; Grade 1, minimal fibrous thickening of alveolar or bronchiolar walls; Grade 2-3, moderate thickening of walls without obvious damage to lung architecture; Grade 4-5, increased fibrosis with definite damage to lung structure and formation of fibrous bands or small fibrous masses; Grade 6-7, severe distortion of structure and large fibrous areas; and Grade 8, total fibrous obliteration of the fields.

\subsection{Hydroxyproline assay}

The measurement of hydroxyproline was conducted with a hydroxyproline measurement kit (Nanjing Jiancheng Bioengineering Institute) according to the manufacturer's instructions. Approximately $30 \mathrm{mg}$ (wet weight) of lung tissue was collected, $1 \mathrm{~mL}$ of alkaline hydrolysate was added, and the tissue was boiled 
at $95{ }^{\circ} \mathrm{C}$ for 20 min with constant mixing. The $\mathrm{pH}$ was adjusted to $6.0-6.8$ using the reagent provided. Approximately 3-4 mL of supernatant was collected for measurement after sorption onto active carbon. The hydrolysate was centrifuged at $3500 \mathrm{rpm}$ for $10 \mathrm{~min}$. Approximately $1.0 \times 10^{-6} \mathrm{~m}^{3}$ of supernatant was then carefully taken for measurement according to the manufacturer's instructions.

\subsection{Flow cytometry}

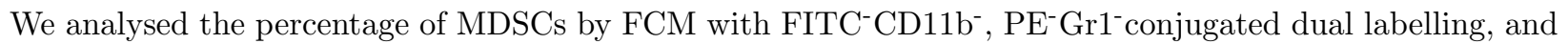
macrophages by $\mathrm{FCM}$ with $\mathrm{APC}^{-} \mathrm{F} 4 / 80^{-}$, $\mathrm{FITC}^{-} \mathrm{CD} 11 \mathrm{~b}^{-}$conjugated dual labelling. After the animals were sacrificed, the lungs of each group were harvested, and collagenase degradation was performed for $2 \mathrm{~h}$. After centrifugation, the cells were stained with different antibodies according to the manufacturer's instructions and then analysed on FCM (Becton-Dickinson, USA) immediately. Finally, the data were analysed using Flow Jo software.

\subsection{Enzyme-linked immunosorbent assay}

TNF- $\alpha$, IL-2, IL-4, IL-10, IL-17A, and IFN- $\gamma$ levels in mouse serum were determined using a Mouse Th1/Th2/Th17 Cytokine Kit (BD Biosciences, Franklin Lake, New Jersey, USA) according to the manufacturer's instructions. Briefy, preparing cytokine standards, cytokine capture microsphere suspensions and serum samples, $5 \times 10^{-8} \mathrm{~m}^{3}$ of capture microsphere suspension, $5 \times 10^{-8} \mathrm{~m}^{3}$ of mouse TH1/TH2/TH17 PE signal antibody, $5 \times 10^{-8} \mathrm{~m}^{3}$ of the corresponding mouse TH1/TH2/TH17 cytokine dilution and $5 \times 10^{-8} \mathrm{~m}^{3}$ of test samples were added in each tube then incubated at room temperature in the dark $3 \mathrm{~h}$. Wash and centrifuge at $200 \mathrm{xg}$ for 5 minutes, discard the supernatant and add $300 \mu \mathrm{L}$ of washing solution to each tube, resuspend the microspheres, analyze the samples using FCM, and analyze the calculation results using BDCBA software.

\subsection{Pharmacokinetics study}

The appropriate amount of YTH-60 was accurately weighed, added to the final volume of 5\% DMSO, 40\% PEG400 and 55\% normal saline, and vortexed or ultrasonicated=to fully mix. Then, 0.4 and $2 \mathrm{mg} \cdot \mathrm{mL}^{-1}$ of clarified dosing solution was used for intravenous administration and oral gavage administration. SD rats were transferred from the Experimental Animal Library (999 M-017), Shanghai Xipuer-Beikai Experimental Animal Co. Ltd. After oral, intravenous or intragastric administration, blood was collected by jugular vein or other suitable methods. Approximately $0.20 \mathrm{~mL}$ of each sample was collected, in the presence of heparin sodium as an anticoagulant. After the blood samples were collected, they were placed on ice and centrifuged to separate plasma within $2 \mathrm{~h}$ (centrifugation conditions: centrifugal force $6800 \mathrm{~g}, 6 \mathrm{~min}, 2-8$ ). The collected plasma samples were stored at $-80 \mathrm{deg} C$ before analysis. After analysis, the remaining plasma samples were stored at -80 for a period of one month.

\section{Statistical analysis}

All in vitro experiments were performed at least in triplicate. Results are expressed as mean +- SD or SEM and $P$-values for comparison of two groups were determined by two-tailed Student's t-test. Statistically significant $\mathrm{P}$ values were considered at: ${ }^{*} \mathrm{P}<0.05,{ }^{* *} \mathrm{P}<0.01$ and ${ }^{* * *} \mathrm{P}<0.001$.

\section{RESULTS}

3.1 Design, synthesis, screening, kinase inhibition profile and molecular modelling studies of YTH-60

A total of 550 novel multikinase small-molecule compounds were designed via computer aided drug design (CADD). The 55 lead compounds that ranked in the top $10 \%$ according to values of the Ludi Energy Estimate were synthesized and screened by kinase inhibition assays (data not shown). Among these, (E)-N-(4-(2-(6-(2,6-dichloro-3,5-dimethoxyphenyl)-1H-indazol-3-yl)vinyl)phenyl)-N-methyl-2(4-methylpiperazin-1-yl)acetamide (YTH-60) displays robust kinase and cellular inhibitory activityin vitro . Its structural formula and synthesis are shown in Fig. 1A and Fig. S1. To clarify the inhibitory effects 
of YTH-60 on multiple proteins, an in vitro kinase assay was performed (Table 1). YTH-60 robustly inhibited FGFR1-3, with $\mathrm{IC}_{50}$ values of 4-7 nM. In addition, YTH-60 inhibited VEGFR1-3 and had an $\mathrm{IC}_{50}$ of 61-123 nM and exhibited relatively weak kinase inhibition of PDGFR $\alpha$ and PDGFR $\beta$. In order to further understand the activity of YTH-60 against FGFR1 kinase, we performed molecular docking into FGFR1 kinase. Figure 1B and 1C showed the interaction modes of YTH-60 with the kinase domain of FGFR1 (PDB ID:5UR1) by computer simulation and computer-based molecular docking methods. YTH-60 is observed to form strong hydrogen-bonding interactions with the surrounding amino acid residues such as GLU531, GLU562, LYS566, GLY567, ASP641. YTH-60 forms hydrophobic interactions with the surrounding amino acid residues such as LEU484, VAL492, ALA512, LYS514, ILE545, VAL561, ALA564, LEU630 and ALA640. In addition, LYS514 of YTH-60 forms an electrostatic force. The above results speculate that YTH-60 could bind with protein stably.

\subsection{YTH-60 inhibits fibroblast activation and proliferation}

To further confirm the effects of YTH-60, we investigated the time- and concentration-dependent effects of YTH-60 on the proliferation of HPF and NIH-3T3 fibroblast cell lines. The viability of these cell lines after exposure to YTH-60 for 24-72 h decreased with increasing concentration and duration of exposure (Fig. $2 \mathrm{~A}, \mathrm{~B}$ and $\mathrm{C}$ ). The results indicated that YTH-60 inhibited the proliferation of the cells in a time- and concentration-dependent manner. These two fibroblast lines were much more sensitive to YTH-60 than the positive control nintedanib (Fig. S2A and B). The cytotoxicity of YTH-60 in vitro was confirmed using the normal human liver cell line LO2. YTH-60 inhibited the viability of LO2 cells less than nintedanib after a short exposure (24 h) (Fig. S2C).

Real-time qPCR and Western blot analysis further demonstrated the inhibitory effects of YTH-60 on fibroblast activation. NIH-3T3 cells were treated with TGF- $\beta_{1}$ and $2.5 \mu \mathrm{M}$ YTH-60 for $24 \mathrm{~h}$, and co-culture with TGF $\beta_{1}$-treated NIH-3T3 cells increased the mRNA levels of collagen I, $\alpha$-SMA and TGF $\beta_{1}$, while YTH-60 reduced this effect (Fig. 2D, E and F). Furthermore, YTH-60 attenuated the expression of fibrosis-associated $\alpha$-smooth muscle actin ( $\alpha$-SMA) and type I collagen (collagen I) induced by TGF- $\beta_{1}$ stimulation (Fig. $2 \mathrm{G}$ ). Likewise, an immunofluorescence assay was performed to investigate the antifibrotic effects of YTH-60 in NIH3T3 and HPH cells. The expression of $\alpha$-SMA was decreased after treatment with $2.5 \mu \mathrm{M}$ YTH-60 for 24 $\mathrm{h}$ (Fig. $2 \mathrm{H}$ and I). These results indicated that YTH-60 could inhibit fibroblast activation and proliferation.

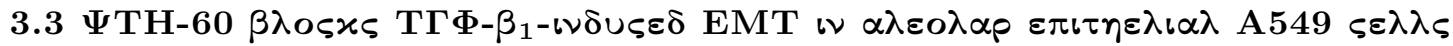

We further evaluated whether the antifibrotic effects of YTH-60 was mediated via inhibition of EMT using an in vitro EMT model. The results indicated that YTH-60 inhibited the proliferation of A549 cells in a time- and concentration-dependent manner (Fig. S3A and B). TGF- $\beta_{1}$-induced morphological alterations in A549 cells were clearly detected, from oval epithelial cells to spindle-shaped fibroblast-like cells (Fig. 3A). YTH-60 intervention markedly blocked the spindle-like mesenchymal morphology of EMT in A549 cells stimulated by TGF- $\beta_{1}$ (Fig. 3B and C). Further study showed that co-culture with TGF $\beta_{1}$-treated A549 cells decreased the mRNA levels of E-cadherin and increased TGF- $\beta_{1}$, while YTH-60 reversed this effect (Fig. 3D and E). Immunofluorescence results further demonstrated that YTH-60 treatment enhanced the expression of E-cadherin compared with that of TGF- $\beta_{1}$ treatment alone (Fig. 3F). YTH-60 treatment increased the expression of the epithelial marker E-cadherin and repressed the expression of the mesenchymal markers $\alpha$-SMA and Vimentin in TGF- $\beta_{1}$-treated A549 cells (Fig. 3G).

\subsection{Pharmacokinetic study of YTH-60 after IV and PO administration to SD rats}

To evaluate the efficacy of YTH-60 in animal models, we first examined the pharmacokinetic profile of YTH60. Blood concentration-time curve of the compound after oral administration and blood concentration-time curve of the compound after intravenous infusion are shown in Fig. S4. After an IV dose, the mean halflife was $4.92 \mathrm{~h}$, and the mean $\mathrm{AUC}(0-\mathrm{t})$ was $772.62 \mathrm{~h} \cdot \mathrm{ng} \cdot \mathrm{mL}^{-1}$. The mean bioavailability was $17.86 \%$ for $20 \mathrm{mg} \cdot \mathrm{kg}^{-1}$ YTH-60 by oral administration. The mean half-life was $8.03 \mathrm{~h}$, and the mean AUC (0-t) was $1379.73 \mathrm{~h} \cdot \mathrm{ng} \cdot \mathrm{mL}^{-1}$ (Table 2). While, the mean bioavailability was $11.9 \%$ for $50 \mathrm{mg} \cdot \mathrm{kg}^{-1}$ nintedanib by oral administration(Roth et al.,2015). This result suggests that YTH-60 might be a potential drug candidate 
with low toxicity and has better absorption than nintedanib.

\subsection{YTH-60 alleviates BLM-induced pulmonary fibrosis in mice}

We induced lung fibrosis by single intratracheal administration of bleomycin(BLM). To investigate the antifibrotic potential of YTH-60, mice were challenged with BLM $\left(2 \mathrm{mg} \cdot \mathrm{kg}^{-1}\right)$ and treated with two different doses of YTH-60 (15 and $\left.30 \mathrm{mg} \cdot \mathrm{kg}^{-1}\right)$ and the positive control nintedanib $\left(30 \mathrm{mg} \cdot \mathrm{kg}^{-1}\right)$ (Fig. 4A). When compared to the control group, bleomycin enhanced pulmonary inflammatory responses and destroyed lung architecture, with thickened alveolar septa and collapsed alveolar spaces, as evidenced by semiquantitative analysis using fibrotic Ashcroft histological evaluation of H\&E-stained lung sections (Fig. 4B, C). Additionally, mice treated with bleomycin displayed notable deposition of collagen, as indicated by not only quantitative histological collagen morphometry assessed by the fibrosis fraction of Masson's trichrome-stained tissues but also biochemical collagen quantification determined by measuring hydroxyproline (Fig. 4B and E). Importantly, these BLM-induced inflammatory and fibrotic features were obviously alleviated after YTH-60 administration in BLM-treated mice (Fig. 4B, C, D and E). Similar findings were obtained when comparing treatment with YTH-60 and nintedanib. In addition, we did not observe obvious lesions in the heart, liver, spleen, or kidneys by microscopic examination (Fig. S5). These data indicate that YTH-60 may protect against lung fibrosis, especially in the early inflammatory phase.

Next, we further investigated the antifibrotic effects of YTH-60. We assessed endogenous mRNA levels of TGF- $\beta_{1}$ in BLM-treated mice. Upregulated $\alpha$-SMA, collagen I and TGF- $\beta_{1}$ in BLM-challenged mice was reduced by administration of YTH-60 (Fig. 5A, B and C). Immunohistochemistry also showed that YTH-60 significantly decreased the expression of $\alpha$-SMA and collagen I (Fig. 5D). In addition, Western blot analysis further demonstrated that BLM upregulated the protein expression of $\alpha$-SMA and collagen I compared with that of control mice. However, YTH-60 treatment reduced the expression of $\alpha$-SMA and collagen I compared with that of BLM-treated mice (Fig. 5E), indicating the inhibitory effects of YTH-60 in bleomycin-induced fibrosis.

\subsection{YTH-60 possesses an anti-inflammatory function in BLM-induced lung fibrosis}

We analysed the influx of important inflammatory cells into the lungs by analysing bronchoalveolar lavage fluid (BALF) in BLM mice on day 14 after BLM instillation. Increased infiltration of WBCs, BASO and LYMPH in the lungs of BLM mice (Fig. 6A, B and C) was consistent with previous observations of elevated lung inflammation in BLM mice through day 14 after BLM treatment(Hao, Cohen, Jennings, Bryson \& Kaplan,2000). YTH-60 significantly reduced the number of infiltrated cells in the BALF of BLM-treated mice. Crosstalk between cytokines and fibroblasts during lung fibrosis is the focus of growing research interest(Borthwick, Wynn \& Fisher). Hence, in this study, we sought to examine the expression of a variety of inflammatory cytokines, such as tumour necrosis factor IFN- $\gamma$ interleukin (IL)-2, IL-4, IL-10, and IL-17A. Marked increases in the production of these inflammatory cytokines were observed on day 14 after BLM exposure compared with those of control mice. However, YTH-60 reduced the expression level of these inflammatory cytokines in the serum of BLM-treated mice (Fig. 6D-I).

\subsection{YTH-60 inhibits bleomycin-induced imbalance in the lungimmune microenvironment}

The cells of the immune system show a rich and multifaceted contribution to IPF, including monocytes, macrophages, myeloid suppressor cells and innate lymphoid cells(Omkar et al.,2018). $\mathrm{M}_{1}$ macrophages are involved in the inflammatory and fibrotic stages of IPF(Murray \& Peter,2015). Activated $\mathrm{M}_{2}$ macrophages produce and secrete proteins such as arginase- 1 and various growth factors and participate in anti-inflammation, tissue repair, collagen synthesis, and ECM deposition(Pesce et al.,2018). There is evidence suggesting that MDSC accumulation is a result of chronic inflammation and the cytokines produced as a result of this inflammation(Ko, Bukowski \& Fincke.). In this study, we treated mice with bleomycin for 14 days, collected lung tissue, and detected the number of macrophages $\left(\mathrm{F} 4 / 80^{+} \mathrm{CD} 11 \mathrm{~b}^{+}\right)$and $\mathrm{MDSCs}\left(\mathrm{CD} 11 \mathrm{~b}^{+} \mathrm{Gr} 1^{+}\right)$by FCM. The results showed that those cells in the bleomycin group were significantly higher than those in the control group, and the number of these cells decreased after YTH-60 treatment and was lower than that in the positive control nintedanib group (Fig. 7A-D). 


\section{DISCUSSION}

Pulmonary fibrosis is a chronic inflammatory condition that is associated with fibroblast proliferation, activation of extracellular matrix synthesis, and abnormally increased collagen. Although the mechanism is unclear, most studies have shown that the increased proliferation of fibroblasts is the most direct cause of fibrosis(Gauldie, Kolb \& Sime.). In addition, epithelial cell injury and repair studies have shown that alveolar type II epithelial cells play an important role in the occurrence of pulmonary fibrosis. Damage to epithelial cell activation of innate immunity can induce the differentiation of myofibroblasts(Tan, Dagher, Hutton \& Bourke,2010). The transformation of epithelial cells into mesenchymal cells is increased(Felton, Borok \& Willis). However, many histological studies have shown that the role of the EMT in pulmonary fibrosis is inconsistent, mainly due to the lack of effective methods to detect the dynamic and transient states of EMT and the lack of markers that clearly assess the interstitial phenotype(Morbini, Inghilleri, Campo, Oggionni, Zorzetto \& Luisetti,2011; Yamada et al.,2008).

In this study, we developed YTH-60, a new multitarget inhibitor, and further studied its functional characteristics and possible anti-pulmonary fibrosis mechanism. Earlier kinase activity assays showed that YTH-60 has potential inhibitory effects on a variety of kinase activities, such as those of FGFR1-4, VEGFR1-3 and PDGFR. We used the clinical drug nintedanib as a positive control and found that YTH-60 has an improved inhibitory effect on a range of fibroblast cell lines and YTH-60 might has better absorption than nintedanib. Here, we demonstrated the effects of YTH-60 against lung fibrosis with invivo and in vitro studies.

In vitro, MTT experiments showed that YTH-60 inhibited the proliferation of a variety of fibroblast cell lines in a concentration-dependent and time-dependent manner, especially in NIH-3T3 cells. TGF- $\beta_{1}$ is well known to initiate and maintain the EMT in various biological systems, including alveolar epithelial cells(Biernacka, Dobaczewski \& Frangogiannis,2011). It is also believed that $T G F \beta_{1}$ secreted by macrophages serves as a paracrine stimulus to fibroblasts to promote the transformation of fibroblasts into myofibroblasts(Wang et al.,2013). Therefore, we used TGF- $\beta_{1}$-stimulated fibroblasts and A549 cells, and YTH-60 effectively slowed the expression of pulmonary fibrosis-related proteins and mRNA. YTH-60 induced the upregulation of epithelial markers and the downregulation of mesenchyme, indicating that YTH-60 may be involved in targeting the EMT process in pathogenesis.

In vivo, we demonstrated that YTH-60 has a good preventive effect on bleomycin-induced pulmonary fibrosis in mice. Bleomycin is known to induce lung damage that results in an acute inflammatory response, followed by fibrotic changes that can replicate many pathological features of human pulmonary fibrosis(Jenkins et al.,2017). Although whether the bleomycin-induced pulmonary fibrosis model can simulate IPF is uncertain, this model has been widely used in the study of pulmonary fibrosis in recent years. More researchers believe that this animal model is a good model because it can be easily replicated, and it involves many aspects that are similar to IPF, such as IPF early-stage alveolitis(Biernacka, Dobaczewski \& Frangogiannis).

Since BLM induces influx of inflammatory cells into the lung during the early inflammatory phase(Jun et al.,2017), intratracheal administration of bleomycin induces acute alveolitis and interstitial inflammation, as defined by the continuous recruitment of leukocytes. White blood cells, such as macrophages and lymphocytes, play an important role in responding to external stimuli and participating in inflammation and tissue remodelling. On day 14 after bleomycin injury, BLM mice showed increased infiltration of inflammatory cells and production of pro-inflammatory cytokines. YTH-60 significantly reduced the number of white blood cells, eosinophils and lymphocytes in BALF.

The increased expression of some inflammatory cytokines in lung tissue is related to the recruitment of inflammatory cells and the accumulation of extracellular matrix components. Our findings also suggested that YTH-60 attenuates collagen accumulation in lung tissue, which may be a follow-up result of inhibiting lung inflammation and lung injury. This may partly explain why inflammation in the lungs is reduced. Therefore, inhibiting leukocyte recruitment directly affects inflammation and tissue repair, which may partially explain the preventive effects of YTH-60 on BLM-induced pulmonary fibrosis. We observed that these proinflammatory cytokines were enhanced in mouse serum on day 14 after bleomycin treatment, and YTH-60 
effectively prevented BLM-induced cytokine secretion. The anti-inflammatory effect of YTH-60 was confirmed by histological analysis, and the semi-quantitative and quantitative evidence in H\&E-stained lung tissue demonstrated the anti-inflammatory effects of YTH-60. In addition, YTH-60 significantly reduced the number of macrophages and MDSCs in mouse lung tissue and then reduced the expression of fibrosis-related proteins and mRNA of $\alpha$-SMA, vimentin and collagen I.

Therefore, we hypothesize that YTH-60 effectively improves pulmonary fibrosis, in part because YTH-60 reduces the expression and activation of some inflammatory cytokines and fibrotic cytokines, thereby reducing multiple interactions between them. However, the detailed mechanism needs further research, and related signalling pathways will also be our next work. In summary, although only the preventive effect of YTH-60 has been evaluated, our findings also suggest a potential treatment for pulmonary fibrosis, and this compound is expected to provide a clinical strategy for idiopathic pulmonary fibrosis in the future.

\section{ACKNOWLEDGEMENTS}

This work was supported by National Natural Science Foundation of China (81500054), the National Science \& Technology Major Project "Key New Drug Creation and Manufacturing Program", China (2019ZX09201001, 2018ZX09711001-011), Shanghai Pharmaceuticals Holding Co.,ltd., The Clinical Research Innovation Project, West China Hospital, Sichuan University (2019HXCX05). We thanks to Medicilon for providing in vivo pharmacokinetic studies.

\section{CONFLICT OF INTEREST}

The authors declare that they have no known competing financial interests or personal relationships that could have appeared to influence the work reported in this paper.

\section{AUTHORS CONTRIBUTION}

Hongyao Liu, Xiuli Wu, Zhihao Liu and Tinghong Ye participated in research design. Hongyao Liu, Xiuli Wu, Cailing Gan, Wei Wei, Xingping Su, Liqun Wang and Lin Yue conducted experiments. Hongyao Liu, Xiuli Wu, Qianyu Zhang, Zui Tan and Tinghong Ye performed data analysis. Yuqin Yao, Liang Ouyang and Luoting Yu provided some reagents and consumables. Hongyao Liu and Xiuli Wu contributed to the writing of the manuscript.

\section{References}

Ashcroft T, Simpson JM, \& Timbrell V Simple method of estimating severity of pulmonary fibrosis on a numerical scale. Journal of Clinical Pathology 41: 467-470.

Biernacka A, Dobaczewski M, \& Frangogiannis NG (2011). TGF- $\beta$ signaling in fibrosis. Growth Factors 29: 196-202.

Borthwick LA, Wynn TA, \& Fisher AJ Cytokine mediated tissue fibrosis. J Biochimica Et Biophysica Acta 1832: 1049-1060.

Datta A, Scotton CJ, \& Chambers RC (2011). Novel therapeutic approaches for pulmonary fibrosis. British Journal of Pharmacology 163:141-172.

Felton VM, Borok Z, \& Willis BC N-acetylcysteine inhibits alveolar epithelial-mesenchymal transition. AJP:Lung Cellular Molecular Physiology 297: L805-L812.

Gauldie J, Kolb M, \& Sime. PJ A new direction in the pathogenesis of idiopathic pulmonary fibrosis? Respiratory Research 3: 1.

Hao H, Cohen DA, Jennings CD, Bryson JS, \& Kaplan AM (2000). Bleomycin-induced pulmonary fibrosis is independent of eosinophils. Journal of Leukocyte Biology 68: 515-521.

Hinz B, Phan SH, Thannickal VJ, Galli A, Bochaton-Piallat ML, \& Gabbiani G (2007). The Myofibroblast

: One Function, Multiple Origins. American Journal of Pathology 170: 1807-1816. 
Ingram J, \& Bonner J EGF and PDGF Receptor Tyrosine Kinases as Therapeutic Targets for Chronic Lung Diseases. Current Molecular Medicine 6: 409-421.

Jenkins RG, Moore BB, Chambers RC, Eickelberg O, Königshoff M, Kolb M, et al. (2017). An Official American Thoracic Society Workshop Report: Use of Animal Models for the Preclinical Assessment of Potential Therapies for Pulmonary Fibrosis. American Journal of Respiratory Cell and Molecular Biology 56: 667-679.

Jun T, A. RG, H. LA, Kurt W, J. ES, Ioanna N, et al. (2017). Exploring Animal Models That Resemble Idiopathic Pulmonary Fibrosis. Frontiers in Medicine 4: 118-.

Karimi-Shah BA, \& Chowdhury BA (2015). Forced vital capacity in idiopathic pulmonary fibrosis-FDA review of pirfenidone and nintedanib. New England Journal of Medicine 372: 1189-1191.

Ko JS, Bukowski RM, \& Fincke. JH Myeloid-derived suppressor cells: A novel therapeutic target. Current Oncology Reports 11: 87-93.

Lin N, Chen S, Pan W, Xu L, Hu K, \& Xu R (2011). NP603, a novel and potent inhibitor of FGFR1 tyrosine kinase, inhibits hepatic stellate cell proliferation and ameliorates hepatic fibrosis in rats. American Journal of Physiology Cell Physiology 301: C469-C477.

Luigi N (2016). Gene therapy returns to centre stage. Nature 526: 351-360.

Masaru, \& Katoh. (2016). Therapeutics Targeting FGF Signaling Network in Human Diseases. Trends in Pharmacological Sciences.

Morbini P, Inghilleri S, Campo I, Oggionni T, Zorzetto M, \& Luisetti M (2011). Incomplete expression of epithelial-mesenchymal transition markers in idiopathic pulmonary fibrosis. Pathology Research \& Practice 207: 0-567.

Murray, \& Peter j (2015). Macrophage activation and polarization. Seminars in Immunology.

Noble PW, Albera C, Bradford WZ, Costabel U, Du BR, Fagan EA, et al. (2016). Pirfenidone for idiopathic pulmonary fibrosis: analysis of pooled data from three multinational phase 3 trials. European Respiratory Journal 47: 243-253.

Noble PW, Carlo A, Bradford WZ, Ulrich C, Glassberg MK, David K, et al. (2011). Pirfenidone in patients with idiopathic pulmonary fibrosis (CAPACITY): two randomised trials. Lancet 377:1760-1769.

Omkar, Desai, Julia, Winkler, Maksym, Minasyan, et al. (2018). The Role of Immune and Inflammatory Cells in Idiopathic Pulmonary Fibrosis. Frontiers in Medicine.

Pesce JT, Ramalingam TR, Mentink-Kane MM, Wilson MS, El Kasmi KC, Smith AM, et al. (2018). Arginase-1-Expressing Macrophages Suppress Th2 Cytokine-Driven Inflammation and Fibrosis. Plos Pathogens 5: e1000371.

Raghu G, Collard HR, Egan JJ, Martinez FJ, Behr J, Brown KK, et al. (2011). ATS/ERS/JRS/ALAT Committee on Idiopathic Pulmonary fibrosis. An official ATS/ERS/JRS/ALAT statement: idiopathic pulmonary fibrosis: evidence-based guidelines for diagnosis and management. American Journal of Respiratory 183.

Richeldi L, Cottin V, Du BR, Selman M, Kimura T, Bailes Z, et al.(2016). Nintedanib in patients with idiopathic pulmonary fibrosis: Combined evidence from the TOMORROW and INPULSIS( $\hat{\mathrm{A}} \mathbb{R})$ trials. Respiratory Medicine 113: 74-79.

Roth GJ, Binder R, Colbatzky F, Dallinger C, Schlenker-Herceg R, Hilberg F, et al. (2015). Nintedanib: From Discovery to the Clinic. Journal of Medicinal Chemistry 58: 1053-1063.

Somia N, \& Verma IM (2000). Gene therapy: trials and tribulations. Nature Reviews Genetics 1: 91-99. 
Tan X, Dagher H, Hutton CA, \& Bourke JE (2010). Effects of PPAR gamma ligands on TGF-beta1-induced epithelial-mesenchymal transition in alveolar epithelial cells. respiratory research 11: 21.

Wang P, Nie X, Wang Y, Li Y, Ge C, Zhang L, et al. (2013). Multiwall Carbon Nanotubes Mediate Macrophage Activation and Promote Pulmonary Fibrosis Through TGF- $\beta$ /Smad Signaling Pathway. Small 9: 3799-3811.

Xu Y (2013). YL529, a novel, orally available multikinase inhibitor, potently inhibits angiogenesis and tumour growth in preclinical models . British Journal of Pharmacology 169.

Yamada M, Kuwano K, Maeyama T, Hamada N, Yoshimi M, Nakanishi Y, et al. (2008). Dualimmunohistochemistry provides little evidence for epithelial-mesenchymal transition in pulmonary fibrosis. histochemistry \& cell biology 129: 453-462.

\section{Hosted file}

Figure.docx available at https://authorea.com/users/323536/articles/452150-yth-60-anovel-multikinase-inhibitor-potently-ameliorates-lung-inflammation-and-fibrosis-inpreclinical-model

\section{Hosted file}

Tables.docx available at https://authorea.com/users/323536/articles/452150-yth-60-anovel-multikinase-inhibitor-potently-ameliorates-lung-inflammation-and-fibrosis-inpreclinical-model 